\title{
Developing a Curriculum for Evolutionary Medicine: Case Studies of Scurvy and Female Reproductive Tract Cancers
}

\author{
Tatjana Buklijas • Felicia M. Low • Alan S. Beedle • \\ Peter D. Gluckman
}

Published online: 14 December 2011

(C) Springer Science+Business Media, LLC 2011

\begin{abstract}
Most early evolutionary thinkers came from medicine, yet evolution has had a checkered history in medical education. It is only in the last few decades that serious efforts have begun to be made to integrate evolutionary biology into the medical curriculum. However, it is not clear when, where (independently or as part of preclinical or clinical teaching courses) and, most importantly, how should medical students learn the basic principles of evolutionary biology applied to medicine, known today as evolutionary or Darwinian medicine. Most clinicians are ill-prepared to teach evolutionary biology and most evolutionary biologists ill-equipped to formulate clinical examples. Yet, if evolutionary science is to have impact on clinical thought, then teaching material that embeds evolution within the clinical framework must be developed. In this paper, we use two clinical case studies to demonstrate how such may be used to teach evolutionary medicine to medical students in a way that is approachable as well as informative and relevant.
\end{abstract}

Keywords Evolutionary medicine - Curriculum .

Medical education · Case study - Scurvy .

Cancers of female reproductive tract $\cdot$ Medical students

\section{Introduction}

Although many early evolutionary thinkers came from a medical background, evolution has had a checkered history

T. Buklijas $(\square) \cdot$ F. M. Low $•$ A. S. Beedle $\cdot$ P. D. Gluckman Centre for Human Evolution, Adaptation and Disease, Liggins Institute, The University of Auckland,

Private Bag 92019, Auckland, New Zealand

e-mail: t.buklijas@auckland.ac.nz in medical education. In the early nineteenth century, Jean-Baptiste Lamarck and then Geoffroy St Hilaire advocated "unity of composition" that ordered all living organisms into a single chain of being (Appel 1987). But forging a biological link from mollusks to kings contravened contemporary religious dogmas and political ideology. Hence, evolution was taught only in revolutionary France and radical schools, such as the University of London (Desmond 1989). In the more liberal political climate of the 1860s, the success of Darwin's theory of evolution by natural selection was followed by its introduction into medical school courses, especially on the European continent. At the University of Jena, medical students first heard about evolution in their anatomical and zoological courses, taught by the two most fervent Darwinians in Continental Europe, Karl Gegenbaur and Ernst Haeckel (Nyhart 1995). At the Vienna Institute of Zootomy between 1861 and 1890, Carl Bernhard Brühl offered courses in evolution to medical and science students, as well as to the general public (Buklijas 2010). In the 1870s in Britain, Francis Maitland Balfour, the first to teach embryology at Cambridge, used Haeckel's biogenetic law-the idea that embryos of higher species in their development pass through adult stages of lower species - to explain evolution (Blackman 2006). Yet by the end of the nineteenth century, the medical curriculum was in flux. New experimental sciences such as physiology were vying for space with established clinical and comparative disciplines such as anatomy and zoology. Even evolution's champion, "Darwin's bulldog" Thomas Huxley, who as president of the Royal Society had considerable influence over the content of medical education in this period, believed evolution irrelevant to the problems doctors had to address. The early twentieth-century medical school reforms - in the U.S. instigated by Abraham Flexner's 
report-coincided with a major crisis of Darwin's teaching in the early 1900s. While no one doubted evolution, natural selection as its underlying mechanism was challenged both by neo-Lamarckians and by the emerging geneticists who argued that significantly new forms appeared suddenly by means of random mutation (Bowler 1992). As "hereditarian" views of the body and disease came to dominate "environmental" ones around the turn of the twentieth century, Darwin's theory and Galtonian arguments provided a framework for organizing ideas - and expressing worries-concerning the perceived increase in the frequency of certain diseases in modern environments (Pick 1989; Zampieri 2009). Yet although certain elements of evolutionary biology exercised influence upon medical disciplines such as anatomy, embryology, infectious diseases and immunology, evolutionary biology itself never became part of the curriculum in the same way as, for example, biochemistry. Indeed, even in the most obvious cases of evolutionary processes at work, such as antibiotic resistance, the language of evolutionary biology has been generally avoided (Antonovics et al. 2007). Furthermore, at no stage was evolutionary thought seen as being of value to the practice of public health and clinical medicine.

But attitudes are now changing, and there has been a recent rise in interest in the application of evolutionary biology to understanding the vulnerability of individuals and populations toward disease. This new approach, known as "Darwinian medicine" or "evolutionary medicine," was pioneered by Randolph Nesse and George C. Williams in the 1980s. From its beginning, it has been accompanied by calls for the inclusion of evolution in medical education (Williams and Nesse 1991). Their argument was that understanding evolution was useful for medical students, practitioners and scientists because it complemented the answers to the proximate questions that biomedical and clinical disciplines asked ("What is the mechanism of disease?") with ultimate answers ("Why and how has the mechanism evolved?"). For example, while the proximate cause of scurvy is lack of vitamin $\mathrm{C}$ in the diet, the ultimate cause is the loss of the ability in humans to synthesize this nutrient. This integrated approach to the human body and human disease would help students bring together anatomical and physiological facts into a coherent framework, open research avenues, and offer a new tool for explaining disease to patients.
Early inquiries into the understanding of and interest in evolutionary knowledge among medical school faculty yielded discouraging results. A 2003 survey found that fewer than half of 50 deans of North American medical schools saw evolutionary biology as important knowledge for physicians; only three schools required a course in evolutionary biology as prerequisite for matriculation, two offered a course or lecture sequence focused on evolution, and eight had any medical school faculty with a primary specialty in evolutionary biology (Nesse and Schiffman 2003). But recent years have seen changes. The American Association of Medical Colleges recently released a report establishing evolutionary biology as a premedical competency alongside other foundations such as chemistry and physics, and pointing to the value of evolutionary approaches within the medical curriculum itself (AAMC-HHMI Scientific Foundation for Future Physicians Committee 2009). The first designated textbook of evolutionary medicine was recently published; it explicitly considers what components of evolutionary medicine have a place in a formal medical curriculum (Gluckman et al. 2009). The book provides a framework for clinicians to understand how evolutionary processes contribute to symptomatology and disease vulnerability. Several medical schools in Europe, Australasia, South America, and the U.S. are now introducing modules on evolutionary medicine into their core curricula.

While there is a growing recognition that evolutionary biology should be taught to medical students, it is not clear at all how it should be done. In particular, how should basic evolutionary principles be made comprehensible to medical students and doctors who were last introduced to evolution in secondary school or undergraduate colleges (if at all) and who have little time or interest for theoretical literature? In this paper, we suggest an approach to the introduction of the principles of evolutionary medicine (Box 1) using diseases/groups of conditions with which students have become acquainted in their preclinical and clinical study. This approach is relevant irrespective of whether the medical course follows an undergraduate degree or not and irrespective of whether the school uses a discipline- or a case-based curriculum.

Both case studies, which can serve as exemplars for additional cases, follow a standardized format in several sections: proximate mechanisms, affected population, 
Box 1. Fundamental principles of evolutionary medicine (based on Box 11.1 from Gluckman PD, AS Beedle and MA Hanson (2009) Principles

of Evolutionary Medicine. Oxford: Oxford University Press. 259)

1. The mature phenotype is the result of genomic and non-genomic inheritance and the cumulative effect of the developmental environment, and is further influenced by the current environment.

2. The history of humans as a species influences our susceptibility to disease. Human history here refers to the influences of drift, bottlenecks and migrations (including founder effects). Genetic drift refers to random demographic effects in small populations that may eliminate or fix rare alleles, whether they are neutral, favored or deleterious. A bottleneck is an event that reduces population size and therefore its genetic diversity. A founder effect refers to the isolation of a small population (for instance by migration) that reduces genetic diversity.

3. Selection operates by maximizing reproductive success of individuals.

4. Selection does not operate to maximize health or longevity.

5. Humans now live in different ways and in different environments to those where most selective processes affecting the modern human phenotype operated.

6. Constraints on evolutionary processes in the presence of environmental novelty can lead to ill health.

7. Definitions of normality/pathology are influenced by the environmental context of the individual and individual variation in phenotype.

8. There is no preordained plan, purpose or design to evolution.

first detailed evolutionary history and ultimate explanations and then the pathways by which evolutionary processes can affect disease risk that this example illustrates (Box 2). The first example/case study that we chose is scurvy, as a well-known, relatively simple deficiency disease. The second example of female reproductive cancers encompasses a complex set of physiological and pathological phenomena resulting from the activity of sex hormones upon bodily tissues. Taken together, they illustrate how evolutionary principles can be explained in an accessible and clinically relevant manner, and how evolutionary biology contributes to the understanding of disease.

Box 2. Pathways by which evolutionary processes can affect disease risk (based on Box 11.2 from Gluckman PD, AS Beedle and MA Hanson (2009) Principles of Evolutionary Medicine. Oxford: Oxford University Press. 260)

1. An evolutionarily mismatched or novel environment: the individual has been exposed to an environment beyond their evolved capacity to adapt, or to an entirely novel environment or challenge.

2. Factors associated with life history strategies: life history strategies are the differing ways in which different species optimize their fitn ess by allocating energy to growth, reproduction and maintenance.

3. Excessive or uncontrolled defense mechanisms: evolved processes which are normally adaptive (for example, fever or immune responses) may in excess become harmful to the individual.

4. Losing the evolutionary arms race against other species, which for humans in the modern environment are in particular micro-organisms.

5. Results of evolutionary ('design') constraints

6. An apparently harmful allele is maintained by balancing selection, where heterozygotes for the 'harmful' allele have a selective advantage over homozygotes for either the 'healthy' or 'harmful' alleles (for example, the malaria-associated haemoglobinopathies such as sickle cell disease).

7. Sexual selection and competition and their consequences.

8. The outcomes of demographic history, including the influences of drift, bottlenecks, migrations and founder effects. 


\section{Case I: scurvy}

\section{Proximate mechanism}

Scurvy is a multi-system disorder most commonly manifested as inflammation and bleeding of gums, skin, bones, joints and digestive tract; keratosis of hair follicles ("corkscrew hair"); anemia, sometimes leading to congestive heart failure; and nonspecific symptoms such as fatigue ( $\mathrm{Li}$ et al. 2008). It is caused by dietary deficiency of vitamin $\mathrm{C}$ (ascorbic acid), found in all fresh fruit and vegetables. The total body pool of vitamin $\mathrm{C}$ is normally about $1,500 \mathrm{mg}$, and at levels below $350 \mathrm{mg}$, which equals elimination of vitamin $\mathrm{C}$ from the diet for 60 to 90 days, symptoms of scurvy will appear. The treatment is repletion of ascorbic acid, which normally leads to visible improvement within days.

Vitamin $\mathrm{C}$ is involved in the hydroxylation of proline to hydroxyproline and lysine to hydroxylysine, necessary steps in the formation of collagen, an essential structural component of the skin, blood vessels, tendons, ligaments and bone. Vitamin $\mathrm{C}$ also has roles in the synthesis of peptide hormones, norepinephrine, and carnitine, in tyrosine metabolism, and in disulfide bond formation in hair. It also has antioxidant activity.

\section{Affected population}

Scurvy, a disorder unknown for most of human history, first appeared in the age of extended sea voyages between the sixteenth and eighteenth centuries. Today, the condition may be found in people eating extreme diets lacking in fruit and vegetables (the homeless, psychiatric patients) or those suffering from gastrointestinal conditions, where the absorption of vitamin $\mathrm{C}$ is hindered (Khonsari et al. 2005).

Evolutionary history, ultimate explanation, and demonstration of evolutionary principles

The majority of vertebrates are able to synthesize ascorbic acid from glucose (Chatterjee et al. 1975). The last step in the pathway requires the enzyme L-gulonolactone oxidase (GULO), present in almost all organisms with the exception of some fish, bird, and mammalian species. All the cases where the species cannot synthesize ascorbate are caused by mutations in the GULO gene.

Among mammals, inability to synthesize vitamin $\mathrm{C}$ is found mostly in one of the two major primate suborders, the Anthropoidea (Haplorrhini): tarsiers, monkeys, apes, and humans. It also occurs in the Order Rodentia: guinea pigs and capybaras. Until recently, it was also believed that all families of bats (Order Chiroptera) lacked GULO, but this has been undermined by a recent study that showed that some previously unexamined species of bats have the ability to synthesize vitamin $\mathrm{C}$, albeit at rather low levels (Cui et al. 2011).

In humans, the loss of synthetic ability is due to a frameshift mutation in GULO leading to a premature stop codon. The presence of different mutations in different species confirms that this gene has mutated a number of times, and in some species the remnants of the gene are more recognizable, while in others no traces can be found. In some passerine bird species, differing mutations that are not clearly related suggest the likelihood of multiple mutational events. In contrast, fruit-eating bats share a common mutation suggesting common ancestry. It seems, based on the current phylogeny of bats, that they have reacquired this ability after losing it once, rather than retaining it throughout (Drouin et al. 2011). Similar reactivation events have also been proposed for those passerine bird species that exhibit synthetic capacity.

The study of the mutation in primates illustrates our phylogenetic history. While the primate suborder of Haplorrhini cannot produce vitamin $\mathrm{C}$ and all share the same mutation, the other primate suborder of non-tarsier prosimians, the Strepsirrhini, retained the synthetic capacity. As these two suborders diverged about 60 million years ago, it is a reasonable presumption that the mutation occurred in an early Haplorrhine frugivore ancestor species.

The currently accepted hypothesis is that these mutations were originally neutral, meaning that deletion of the vitamin $\mathrm{C}$ production pathway had no selective advantage or disadvantage. All the mutations are located precisely in GULO, where they affect the ability to make vitamin $\mathrm{C}$ only, rather than elsewhere in the pathway where they would influence the production of other molecules. All species which have lost the capacity to synthesize vitamin C have diverse, yet consistently vitamin C-rich, diets (Drouin et al. 2011). The daily vitamin C consumption of gorillas in the wild far exceeds the recommended daily allowance for adult humans, $1 \mathrm{mg} / \mathrm{kg} /$ day. So, if ascorbic acid was widely available in the human primitive diet, then selection to retain functioning GULO would have been relaxed (Lahti et al. 2009).

An alternative hypothesis is that losing the capacity to synthesize vitamin $\mathrm{C}$ was advantageous because vitamin $\mathrm{C}$ synthesis leads to the production of hydrogen peroxide and the depletion of glutathione (Bánhegyi et al. 1996). As glutathione is an important antioxidant, and as access to ascorbate remained unhindered, the loss of internal synthesis took place. However, this alternative hypothesis does not account for the reacquisition of vitamin $\mathrm{C}$ synthetic capacity in bats which, were the loss of synthetic capacity advantageous, would have been selected against (Drouin et al. 2011). 
Pathways of altered disease risk illustrated by this example

- Mismatch pathways leading to disease. A mutation that was originally neutral may become biologically significant in a changed environment. Humans normally get adequate amounts of vitamin $\mathrm{C}$ in their diet, and it is only with extreme shifts from normative food sources that scurvy appears. In other words, a mutation that was probably neutral when it first appeared has become pathological when the environment changed into a novel one for that lineage, one without vitamin $\mathrm{C}$ in dietary sources.

\section{Case II: female reproductive cancer}

\section{Proximate mechanism}

Malignant neoplasms of the breast are most commonly derived from the epithelial lining of the ducts and lobules, thus classified as ductal or lobular carcinomas. The susceptibility to carcinogens is directly related to the epithelial cell proliferation rate and inversely related to the degree of tissue differentiation. Full maturation of the breast tissue only occurs after the first pregnancy (Russo et al. 2000), while breastfeeding may allow for the exudation of pre-neoplastic cells.

Malignancies of the ovary are in most cases derived from the ovarian epithelium (although some originate in fallopian tubes); few arise from egg cells or supporting cells. Like in any other cancer, a series of somatic mutations or epimutations is required for the neoplastic potential to be expressed (Greaves 2000).

\section{Affected population}

Breast cancer is the most common malignancy in women, and its incidence has been on the rise in the last several decades (Jemal et al. 2010). Primary risk factors for breast cancer include female gender, age over 50 (peri- and postmenopausal period), nulliparity or no breastfeeding, hormone-replacement therapy, obesity (but only in postmenopausal women), alcohol intake, tobacco exposure, exposure to endocrine disruptors, shift work and lack of exercise (Rossouw et al. 2002; The ESHRE Capri Workshop Group 2011). Diet, especially high animal fat intake, has been suggested but has not been proven as an independent risk factor.

Genetic factors also have an impact on risk, although the most common mutations account for only $2-3 \%$ of breast cancers. Best known is the high (up to $87 \%$ ) risk of lifetime breast cancer in women carrying one of numerous loss-offunction mutations in the BRCA1 and BRCA2 tumor suppressor genes (Thompson et al. 2002; The Breast Cancer Linkage Consortium 1999). Since molecular phylogeny studies suggest that many of the most common alleles at BRCA1 and BRCA2 arose as new mutations in the relatively recent (tens of generations) past (Slatkin and Rannala 2000), the prevalence of these apparently deleterious alleles and their persistence in the population raises some interesting issues. Although most of the excess mortality in carriers of BRCA1 and BRCA2 mutations occurs late in life, after the reproductive period, sufficient cases occur earlier in life such that negative selection might be expected to act to decrease the frequency of the deleterious alleles. That this is not occurring suggests that the mutations also display beneficial (fitness-enhancing) effects in the reproductive period that trade off against their negative (increased cancer susceptibility) effects in later adulthood, a process known as antagonistic pleiotropy. Indeed, a recent study indicated that under natural fertility conditions, women carrying $B R C A 1 / 2$ mutations had markedly increased fecundity (and, as expected, increased post-reproductive mortality) compared with controls (Smith et al. 2011). The increased fecundity was actualized by decreased interbirth interval and a longer reproductive period (greater age at last birth); the proximate (molecular) mechanisms relating altered function of BRCA1/ 2 to increased fecundity remain unclear.

While ovarian cancer is much less frequent than breast cancer, with an incidence rate of around 3\% in the female population, the high mortality associated with this disease makes it one of the top five causes of cancer death among women in many developed countries (Jemal et al. 2010). Primary risk factors include age, nulliparity or no breastfeeding, no history of oral contraceptives, history of hormone-replacement therapy, and obesity. Genetic factors also play a role, as women with a first-degree relative who has developed the disease have a greater risk, which increases further when two or more relatives have been affected. Furthermore, BRCA1 and BRCA2 mutations are associated with an increased risk of ovarian cancer; mutations in these genes confer a lifetime risk of ovarian cancer of up to $66 \%$ (Thompson et al. 2002; The Breast Cancer Linkage Consortium 1999).

Evolutionary history, ultimate explanation, and demonstration of evolutionary principles

Epidemiological evidence indicates that late menarche, early first birth, high parity, early menopause, prolonged lactation reduce the risk of breast as well as ovarian cancer (Eaton et al. 1994). While all of these features characterized women in the Paleolithic as well as modern day huntergatherers, modern Western women have early menarche, a large gap between menarche and first birth, low parity, and relatively short lactation. 
While the postponement of reproduction, low parity, and short lactation are outcomes of social, economic, and political developments that began in the nineteenth century, lowering of the age at menarche is a phenomenon that needs explanation from the evolutionary perspective. In the developed world, the age at menarche has fallen from 17 in the nineteenth century to about 12.5 years in the late twentieth century (Whincup, Gilg et al. 2001; Parent et al. 2003; Gluckman and Hanson 2006; Papadimitriou et al. 2008). It has been suggested that improved nutrition played a major role in this phenomenon. Change in the age at menarche is one of the life history-associated set of mechanisms that shows plasticity due to environmental modulation (see Box 2, Pathway 2). A child exposed to placental insufficiency or poor nutrition in utero may anticipate a hostile external environment, sometimes including early death and consequently trade off intrauterine growth for survival to birth and (eventually) early puberty. At the same time, a fetus and child fed plentifully is able to increase fitness by extending the length of the reproductive period in both directions. So both underfed and well fed children start menarche early (Parent et al. 2003; Sloboda et al. 2007; Papadimitriou et al. 2008). Yet, in later life, the length of the reproductive period as well as the physiological markers of reproductive function markedly differs (Jasienska et al. 2006a, b). Indeed, a recent study of natural selection in a contemporary population has found that the age at menopause is increasing (Byars et al. 2010).

The increased age at first birth, low number of births, and short breastfeeding are all part of change in reproductive behavior that began in the West with the demographic transition about two centuries ago, but is today underway in many parts of the world, in particular in East Asia (Frejka and Sardon 2004; Frejka et al. 2010). Hunter-gatherers with menarche at 16 , average first birth at about 19.5, breastfeeding for the first three or four years of the child's life, an average completed family size of five or six live births, and menopause at around 47 years of age, experience only about 188 ovulations (Eaton et al. 1994). Among the Dogon of Mali, a traditional farming West African population with a mean of $8.6 \pm 3$ live births per woman, women aged 20-34 years had a median of only two menses each over the two-year study period. Their median number of menses per lifetime was just 100 (Strassmann 1999). Modern women, by contrast, have menarche at 12.5, first birth at 24-27 years of age, and a completed family size of 1.8 , so by the age of menopause at 47.5 they may experience close to 500 ovulations (Eaton et al. 1994). The high number of ovulations, it has been argued, mechanically injures the ovarian epithelium while exposing it to locally high hormonal levels, and so increases the risk of ovarian cancer. The reduction of the ovarian cancer risk associated with oral contraceptive use is probably at least partly explainable by ovulation suppression (Siskind et al. 2000). With regard to breast cancer, the lack of full maturation of breast tissue in nulliparous women as well constant proliferative stress on the ductal and lobular epithelium by estrogen and progesterone leads to higher rates of carcinogenesis. The use of hormone-replacement therapy, shown to increase the risk of breast cancer, extends the exposure to estrogen and progesterone (Rossouw et al. 2002). At the same time, reduced lactation also removes the beneficial loss and renewal of ductal epithelial cells by lactational "washout".

Pathways of altered disease risk illustrated by this example

- An evolutionarily mismatched or novel environment. The marked change in human reproductive behavior, involving the use of contraception and hormonereplacement therapy, nulliparity, few and late pregnancies as well as reduced lactation, has created a novel female endocrine environment that differs from the environment in which we evolved, an environment of pregnancy and/or lactational amenorrhea for the majority of fertile years. The novel environment has exposed vulnerability to the carcinogenic effects of sex hormones. The loss of the protective mechanisms associated with pregnancy, lactational amenorrhea, and washout as well longer lifetime exposure to ovulating ovaries has allowed potentially damaged cells to be retained and turn malignant.

- Life history and trade-off associated factors. The fall in the age at menarche, driven by improved nutrition, has extended the potential reproductive period, yet it has also increased the time that the breast and the ovarian epithelia are exposed to the mechanical and endocrine effects of ovulation. Furthermore, because the human life span is now much longer and there is an age-related linear increase in the risk of cancer detected in postmenopausal women, the total lifetime risk of cancer becomes much larger. Finally, the effects of BRCA1/2 mutations on fecundity and cancer susceptibility provide an excellent example of antagonistic pleiotropy, trading off increased reproductive fitness against impaired somatic maintenance (tumor suppression).

- Selection operates by maximizing fitness. For all cancers, neoplastic progression represents a Darwinian process of mutation, variation, and selection among cell lineages within individuals, such that tumor cell lineages with enhanced survival or proliferation capacity will outcompete less "fit" lineages. Such microevolutionary factors play a role in the development of 
resistance to cancer therapy, and understanding the selection pressures and trade-offs involved may lead to new treatment approaches.

\section{Conclusion}

To medical students, evolutionary principles may seem dry and distant from their interests and from the rest of the medical school curriculum. Yet by engaging students' attention with case studies of diseases familiar from other subjects, we can introduce them to an evolutionary way of thinking in a way that is both attractive and educational. The discussed case studies have shown how two very different disease categories, a rare vitamin deficiency and relatively frequent reproductive cancers, may be used to explain evolutionary concepts and principles and to elucidate one or more pathways to disease development. The case of scurvy illustrates the importance of the phylogenetic approach ("tree-thinking") as well as showing how a change of nutritional environment exposes a hitherto hidden predisposition to disease. The case of reproductive cancers vividly shows how life history-associated factors, along with changes in reproductive environment and demography, dramatically alter disease risk.

This approach, we suggest, creates a framework for evolutionary thought to become pervasive in the medical curriculum. Ultimate explanations are of considerable value in the patient-doctor interaction. Similarly, in public health a more explicit understanding of evolutionary principles is of value. We are currently preparing a library of such case studies, which will be published either as an online resource or a book, which will include examples of most disease categories demonstrating all of the pathways listed in Box 2. We hope that this library will find broad use among teachers of evolutionary medicine and their students.

Acknowledgments We thank the National Research Centre for Growth and Development (New Zealand) for support.

\section{References}

AAMC-HHMI Scientific Foundation for Future Physicians Committee. Scientific foundations for future physicians. Washington, DC: American Association of Medical Colleges and Howard Hughes Medical Institute; 2009.

Antonovics J, Abbate JL, et al. Evolution by any other name: antibiotic resistance and avoidance of the E-word. PLoS Biol. 2007;5(2):e30.

Appel TA. The Cuvier-Geoffroy debate: French biology in the decades before Darwin. New York: Oxford University Press; 1987.

Bánhegyi G, Csala M, et al. Ascorbate synthesis-dependent glutathione consumption in mouse liver. FEBS Lett. 1996;381:39-41.
Blackman HJ. Anatomy and embryology in medical education at Cambridge University, 1866-1900. Med Educ. 2006;40(3):219-26.

Bowler P. The eclipse of Darwinism: anti-Darwinian evolution theories in the decades around 1900. Baltimore: John Hopkins University Press; 1992.

Buklijas T. Public anatomies in fin-de-siècle Vienna. Medicine Studies. 2010;2(1):71-92.

Byars SG, Ewbank D, et al. Natural selection in a contemporary human population. PNAS. 2010;107 suppl 1:1787-92.

Chatterjee IB, Majumder AK, et al. Synthesis and some major functions of vitamin C in animals. Ann N Y Acad Sci. 1975;258:24-47.

Cui J, Pan Y-H, et al. Progressive pseudogenization: vitamin C synthesis and its loss in bats. Mol Biol Evol. 2011;28(2):1025-31.

Desmond AJ. The politics of evolution: morphology, medicine and reform in radical London. Chicago: The University of Chicago Press; 1989.

Drouin G, Godin J-R, et al. The genetics of vitamin C loss in vertebrates. Curr Genom. 2011;12:371-8.

Eaton SB, Pike MC, et al. Women's reproductive cancers in evolutionary context. Q Rev Biol. 1994;69(3):353-67.

Frejka T, Jones GW, et al. East Asian childbearing patterns and policy developments. Popul Dev Rev. 2010;36(3):579-606.

Frejka T, Sardon J-P. Childbearing trends and prospects in low-fertility countries: a cohort analysis. Dordrecht: Kluwer; 2004.

Gluckman PD, Beedle AS, et al. Principles of evolutionary medicine. Oxford: Oxford University Press; 2009.

Gluckman PD, Hanson MA (2006) Evolution, development and timing of puberty. Trends Endocrinol Metab 2006;17(1):7-12.

Greaves M. Cancer: the evolutionary legacy. Oxford: Oxford University Press; 2000.

Jasienska G, Thune I, et al. Fatness at birth predicts adult susceptibility to ovarian suppression: an empirical test of the Predictive Adaptive Response hypothesis. PNAS 2006a;103(34):12759-62.

Jasienska G, Ziomkiewicz A, et al. High ponderal index at birth predicts high estradiol levels in adult women. Am J Hum Biol. 2006b;18:133-40.

Jemal A, Siegel R, et al. Cancer statistics, 2010. CA Cancer J Clin. 2010;60:277-300.

Khonsari H, Grandiere-Perez L, et al. Le scorbut n'a pas disparu: histoire d'une maladie réémergente. Rev Med Interne. 2005;26 (11):885-90.

Lahti DC, Johnson NA, et al. Relaxed selection in the wild. Trends Ecol Evol. 2009;24(9):487-96.

Li R, Byers K, et al. Gingival hypertrophy: a solitary manifestation of scurvy. Am J Otolaryngol. 2008;29(6):426-8.

Nesse RM, Schiffman JD. Evolutionary biology in the medical school curriculum. BioScience. 2003;53(6):585-7.

Nyhart L. Biology takes form: animal morphology and the German universities, 1800-1900. Chicago: University of Chicago Press; 1995.

Papadimitriou A, Fytanidis G, et al. Age at menarche in contemporary Greek girls: evidence for levelling-off of the secular trend. Acta Paediatr. 2008;97(6):812-5.

Parent AS, Teilmann G, et al. The timing of normal puberty and the age limits of sexual precocity: variations around the world, secular trends, and changes after migration. Endocr Rev. 2003;24(5):66893.

Pick D. Faces of degeneration: a European disorder, c.1848-c.1918. Cambridge: Cambridge University Press; 1989.

Rossouw JE, Anderson GL, et al. Risks and benefits of estrogen plus progestin in healthy postmenopausal women: principal results from the Women's Health Initiative randomized controlled trial. JAMA. 2002;288(3):321-33.

Russo J, Hu YF, et al. Developmental, cellular, and molecular basis of human breast cancer. J Natl Cancer Inst Monogr. 2000;27:17-37.

Siskind V, Green A, et al. Beyond ovulation: oral contraceptives and epithelial ovarian cancer. Epidemiology. 2000;11(2):106-10. 
Slatkin M, Rannala B. Estimating allele age. Annu Rev Genomics Hum Genet. 2000;1:225-49.

Sloboda DM, Hart R, et al. Age at menarche: influences of prenatal and postnatal growth. J Clin Endocrinol Metab. 2007;92(1):4650.

Smith KR, Hanson $\mathrm{H}$, et al. Effects of $B R C A 1$ and $B R C A 2$ mutations on female fertility. Proc R Soc B. 2011. doi:10.1098/rspb.2011.1697.

Strassmann BI. Menstrual cycling and breast cancer: an evolutionary perspective. J Womens Health. 1999;8(2):193-202.

The Breast Cancer Linkage Consortium. Cancer risks in BRCA2 mutation carriers. J Natl Cancer Inst. 1999;91(15):1310-6.
The ESHRE Capri Workshop Group. Perimenopausal risk factors and future health. Hum Reprod Update. 2011;17(5):706-717.

Thompson D, Easton DF, et al. Cancer incidence in BRCA1 mutation carriers. J Natl Cancer Inst. 2002;94(18):1358-65.

Whincup PH, Gilg A, et al. Age of menarche in contemporary British teenagers: survey of girls born between 1982 and 1986. BMJ 2001;322(7294):1095-1096.

Williams GC, Nesse RM. The dawn of Darwinian medicine. Q Rev Biol. 1991;66(1):1-22.

Zampieri F. Medicine, evolution, and natural selection: an historical overview. Q Rev Biol. 2009;84(4):333-55. 